\title{
Potential of Digital Flashing Tomosynthesis for Angiocardiographic Evaluation
}

\author{
Georg M. Stiel, Ludmilla S.G. Stiel, and Christoph A. Nienaber
}

\begin{abstract}
A system for digital flashing tomosynthesis (DFTS) consists of four electrocardiogram-gated and simultaneously flashed $x$-ray tubes, a 14" image intensifier, a unit for digital subtraction angiography (DSA), a personal computer-hosted transputer network for threedimensional (3D) reconstruction as well as for quantitative coronary angiography and ventriculography, a display unit and an individual digital archive. DFTStomoangiograms may be presented in single slice mode or as multiple slices of arbitrary thickness, using rotating and stereographic presentation of 3D images. DFTS represents a configuration for standardized digital angiocardiography with digital archiving and assures optimal reproducibility and safety. This angiographic configuration is feasible for both ambulatory angiography to allow high volume cardiovascular angiographic screening and for the quantitative assessment of natural progression or potential regression of coronary artery disease resulting from interventional or pharmacological therapy.

Copyright 11992 by W.B. Saunders Company
\end{abstract}

KEY WORDS: angiocardiography, screening angiography, digital subtraction, coronary artery disease, tomography, $x$-ray, computed.

D' IGITAL ANGIOCARDIOGRAPHY is widely accepted as a useful clinical and investigative tool that also allows for quantitative assessment of obstructive coronary artery disease (CAD) and regional left ventricular dysfunction. During the past decade, most studies from various institutions have used visual interpretation of coronary angiograms to determine the presence or absence of significant CAD. However, the visual assessment is plagued by a high level of interobserver and intraobserver variability. ${ }^{1}$ The development of quantitative coronary angiography has eliminated most of the difficulties associated with visual interpretation. ${ }^{2}$ Whereas the reproducibility of repeat quantitative measurements is excellent, the re-

From the Division of Cardiology, University Hospital Eppendorf, Hamburg, Germany.

Address reprint requests to Georg $M$. Stiel, MD, MSc, University Hospital Eppendorf, Division of Cardiology, Department of Internal Medicine, Martinistraße 52, D-2000 Hamburg 20, Germany.

Copyright 01992 by W.B. Saunders Company

0897-1889/92/0503-0009\$03.00/0 producibility of repeat coronary angiograms is still limited. Progression and regression of coronary atherosclerosis are judged according to changes in the measurements of stenoses obtained from serial coronary angiograms. A major limitation of serial angiographic studies is that repeated angiograms are subject to variations of parameters such as image angulation, magnification, and contrast density, leading to poor agreement with previous or follow-up studies. Therefore, the reproducibility of repeat coronary angiograms is primarily dependent on the precision and comparability of the angiographic procedure and only secondarily related to the reproducibility of repeat quantitative measurements.

Current and future interventional cardiology demands a valid concept for the quantitative assessment of the early stage of CAD and for the objective demonstration of progression or regression of coronary atherosclerosis resulting from therapeutic interventions with optimal reproducibility ideally using three-dimensional (3D) reconstruction from multiple projections. Computerized quantitative assessment is the only acceptable method to study the efficacy of such interventions, but even this technique has inherent limitations and difficulties related to the interpretation of the angiographic information. Some problems may be solved by a new, patient-defined, standardized, and reproducible configuration of digital angiocardiography as a fundamental prerequisite for angiocardiographic screening in a fully digital envirement of a catheterization laboratory. In addition, it has long been the angiographer's desire to depict the anatomy and morphology of coronary arteries using a $3 \mathrm{D}$ presentation.

However, conventional and computed tomography proved not to be suitable for imaging of the rapidly moving coronary arteries because of the relatively long acquisition time. Flashing tomosynthesis is an innovative concept to avoid these problems. ${ }^{3.5}$ A configuration with 24 and $16 \mathrm{x}$-ray tubes has been tested for best visualization of the cerebral and coronary arteries. ${ }^{6-10}$ 
Digital flashing tomosynthesis (DFTS) represents the latest development of the digital tomographic technique and may evenallow angiocardiographic screening with 3D presentation of coronary lesions, including complete quantitative analysis and individual digital archiving for each patient. ${ }^{11}$

\section{SYSTEM CONFIGURATION}

\section{Outline of the System}

The recording equipment of a DFTS system consists of four electrocardiogram (ECG)-gated $\mathrm{x}$-ray tubes with a rotating anode (Philips SRO 20-55, Philips Medical Systems, Shelton, CT) simultaneously flashed by a $120-\mathrm{kW}$ generator MCM 120) as shown in Fig 1. All tubes are simultaneously powered by a voltage of up to $100 \mathrm{keV}$ to deliver a current of up to $300 \mathrm{~mA}$ per tube. Because no cine acquisition is performed, the heating capacity of this system does not play any significant role. A detector consists of a conventional 14-in x-ray image intensifier (Philips 14-in XII, Philips, Eindhoven, Netherlands) and TV system (2-in Plumbicon DTV-6). This system is directly connected to a digital subtraction angiography (DSA) unit (Philips DVI-II) for direct digitizing. Distortion of the image intensifier is allowed before the reconstruction step. The system has a resolution of $512 \times 512$ pixels with 256 ( 8 bits) shades of gray. The spacial resolution of the system is $0.4 \mathrm{~mm}$. The $\mathrm{x}$-ray tubes are arranged in the corners of a square array. The geometry of the DFTS system is characterized by a focus to object distance of $1,050 \mathrm{~mm}$, an object to image intensifier distance of $150 \mathrm{~mm}$, and a field of view of $80 \times 80$ $\mathrm{mm}$. This is shown in Fig 2. A system with 15-in $x$-ray image intensifier (Philips 15-in XII) and $1,024 \times 1,024 \times 10$-bit matrix is also available. The angle between the central beam of each pair of $\mathrm{x}$-ray tubes is $58^{\circ}$ providing a $30^{\circ}$ right anterior oblique and a $30^{\circ}$ left anterior oblique view. In addition, each $\mathrm{x}$-ray tube allows for a $45^{\circ}$ craniocaudal or caudocranial angulation. The array must be rotated to generate steeper craniocaudal projections. The fifth $\mathrm{x}$-ray tube placed in the center is primarily used for positioning the patient under the DFTS equipment and for placing the catheters under fluoroscopic control. Each x-ray tube can also be selectively used for fluoroscopy. Scatter radiation is re-

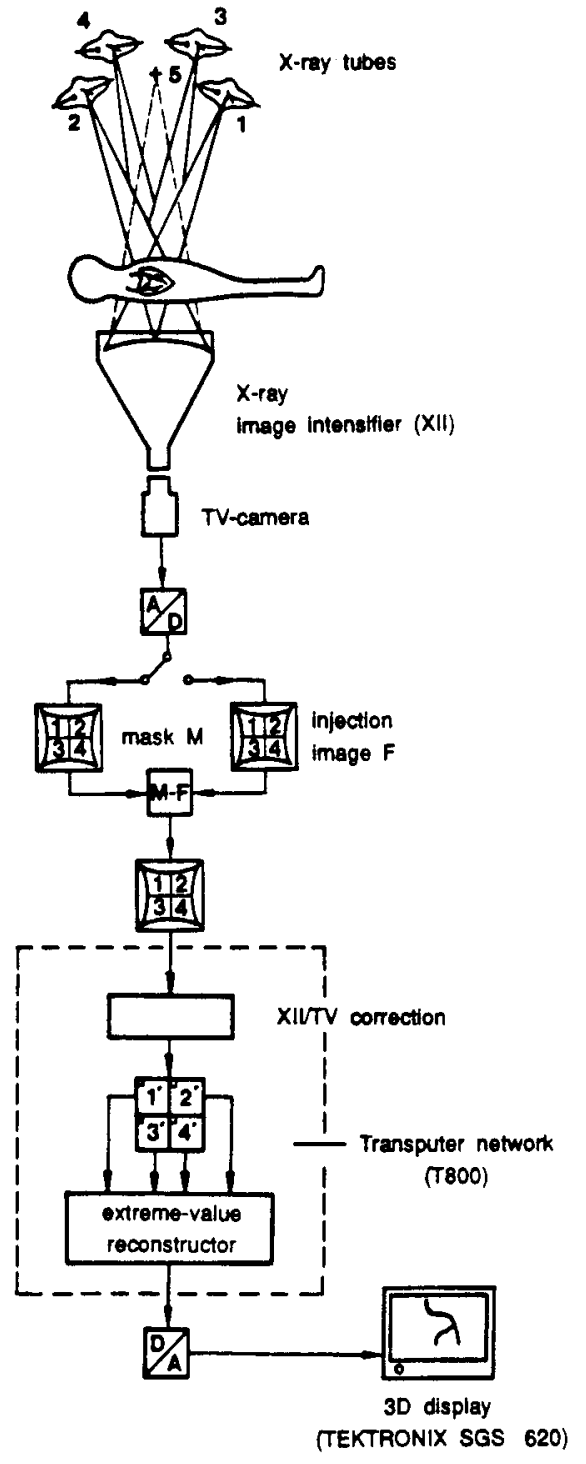

Fig 1. Principle of the DFTS. The recording system consists of four ECG-triggered simultaneously flashed $x$-ray tubes and a 14-inch $x$-ray image intensifier with a TV-chain. The data processing follows against a digital subtraction unit and an extreme-value reconstructor.

duced by an antiscatter grid; the lamellae are adjusted according to the orientation of the corresponding $\mathrm{x}$-ray tubes. ${ }^{9}$

\section{Image Acquisition}

The radiation exposure time of flashing tomosynthesis is less than 40 milliseconds, but varies with the body mass of a given patient. Each ECG-gated $x$-ray flash generates a multiperspective image, ie, the DFTS-multiangiogram as shown in Fig 3. For dynamic studies, a series of ECG-synchronized DFTS-multiangiograms may be generated in any selected phase of the 

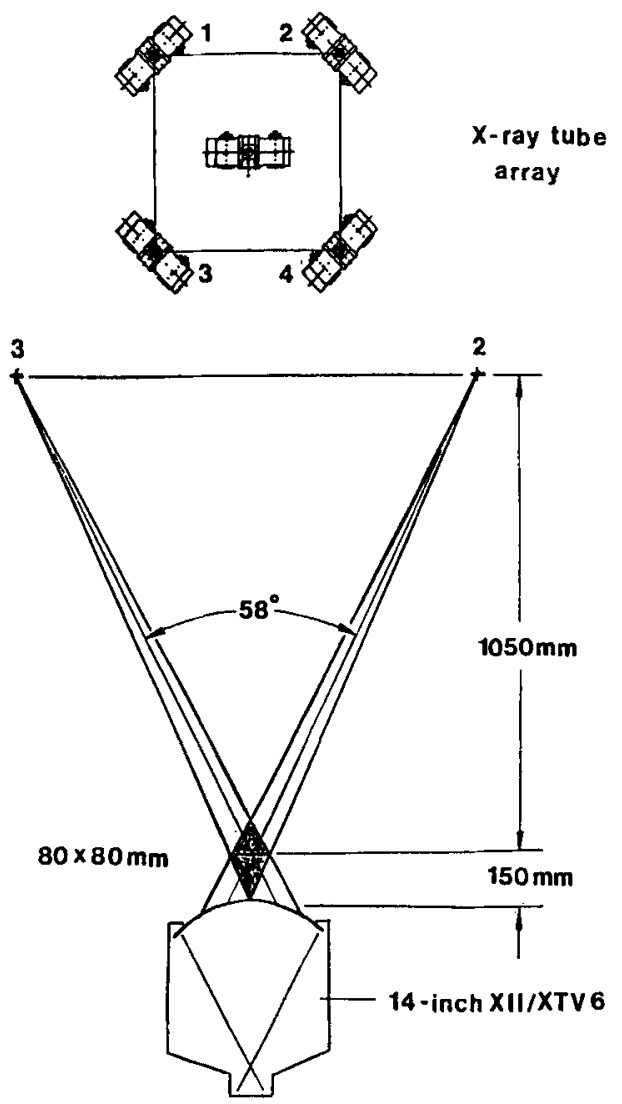

Fig 2. Geometry of the DFTS recording equipment.

cardiac cycle. Each generated DFTS-multiangiogram can be used for the reconstruction of DFTS-tomoangiograms either as a single slice or as multiple slices of arbitrary thickness as

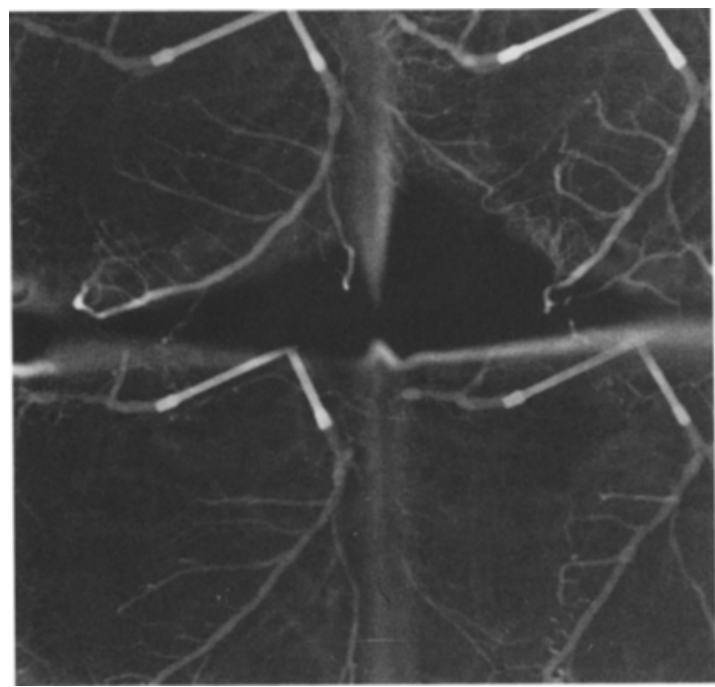

Fig 3. DFTS-multiangiogram as multiperspective image of the left coronary artery. shown in Fig 4. Such DFTS-multiangiograms of a DFTS-multiperspective image or image series may even be used for 3D reconstruction and 3D presentation.

A DFTS-tomoangiogram provides the visualization of the coronary artery tree over a segment of any length. Moreover, the DFTStomoangiogram allows enlargement for both better visual and improved quantitative assessment of the coronary morphology.

For digital subtraction angiography (DSA), an ECG-synchronized DFTS-multiangiogram or a series of DFTS-multiangiograms of the coronary artery tree can be flashed without contrast media to be used for a mask. ${ }^{12}$ However, for preliminary postmortem studies, DSA studies were not performed because a coronary DFTSmultiangiogram without contrast media was not available before contrast filling. Therefore, a "pseudomask technique" was used instead, in which a second image is extracted by a digital filtering technique (selecting of a low-rank gray level) from an original image and silhouettes of blood vessels have been eliminated. ${ }^{13}$ This technique avoids an unsharp mask, where vessels may appear somewhat blurred. Then, the subtraction of these two images show the blood vessel patterns of the original image. This technique represents a digital subtraction process with the original and pseudomask images corre-
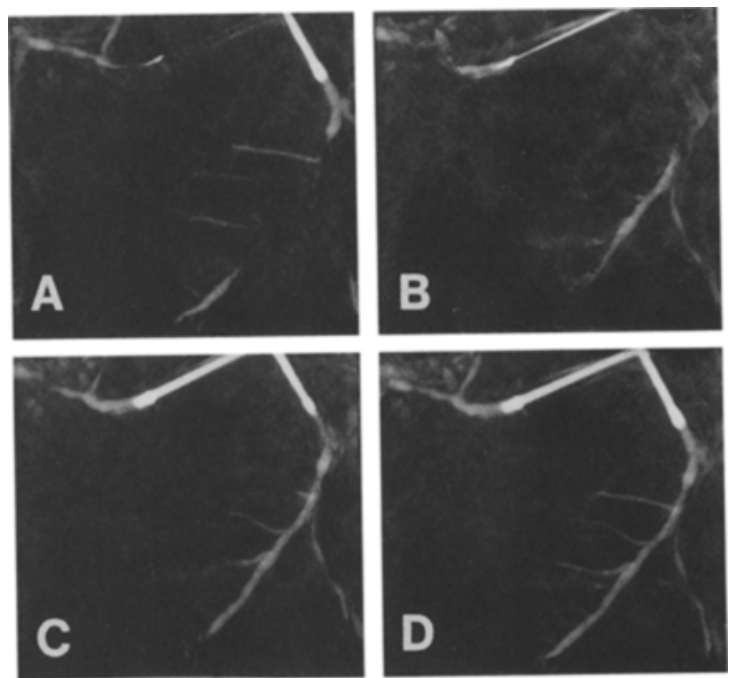

Fig 4. DFTS-tomoangiograms after reconstruction from the multiperspective image of Fig 3 after summing-up of slices 1 to 15 (A), 16 to 30 (B), 31 to 45 (C), and 1 to 45 giving a new synthetic posterior-anterior projection (D). 
sponding to the postinjection and preinjection exposures, respectively. The effect of the pseudomask technique on an x-ray image of a left coronary artery is shown in Fig 5.

\section{Distortion Correction}

The principle of the distortion correction is shown in Fig 6. The spatial coordinates $x_{\mathrm{i}}$ and $y_{\mathrm{i}}$ of a distorted input image have to be mapped onto coordinates $x_{\mathrm{s}}$ and $y_{\mathrm{s}}$ of an undistorted output image. If the distortions are not known beforehand, they have to be determined by imaging a test grid, consisting of equally spaced points in the $x$ and $y$ directions. To allow for a simple pattern recognition, a regular grid with well-known points was chosen. A simple patternrecognition procedure based on threshold and gravity can recognize the mapped grid and automatically associate it pointwise with the original grid. There is no need for selecting reference points. This method offers great flexibility and even considers local distortion factors such as earth magnetism. The gauge step needs not be repeated as long as the parameters of the imaging system remains unchanged.

A polynomial model is applied to describe the functional relation between the mapped grid points and the original grid in the $x$ and $y$ directions:

$$
\begin{array}{r}
x_{i}=f\left(x_{s}, y_{s}\right)=f_{0}+f_{1} y_{s}+f_{2} x_{s}+f_{3} y_{s}^{2}+f_{4} y_{s} x_{s} \\
+f_{5} x_{s}^{2}+f_{6} y_{s}^{3}+f_{7} y_{s}^{2} x_{s}+f_{8} y_{s_{s}} x_{s}^{2}+f_{9} x_{s}^{3} \\
y_{i}=g\left(x_{s}, y_{s}\right)=g_{0}+g_{1} y_{s}+g_{2} x_{s}+g_{3} y_{s}^{2}+g_{4} y_{s} x_{s} \\
+g_{5} x_{s}^{2}+g_{6} y_{s}^{3}+g_{7} y_{s}^{2} x_{s}+g_{8} y_{s} x_{s}^{2}+g_{9} x_{s}^{3}
\end{array}
$$

The coefficients of the polynomials are determined by a least-squares fit. The mapped grid points will be matched with the required pixel accuracy. The geometric position of all ele-
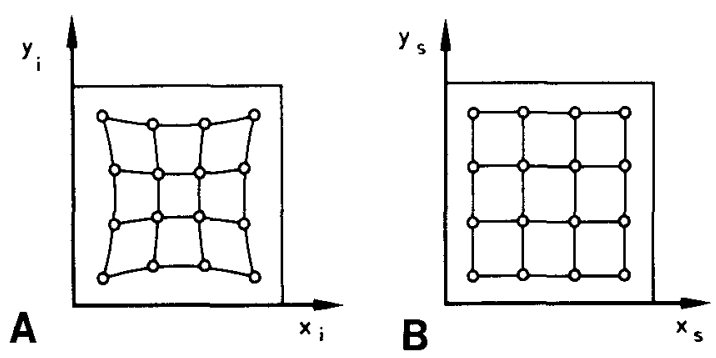

Fig 6. Geometrical principle of distortion correction: distorted input image (A) and undistorted output image (B).

ments of the image matrix are computed and stored in a special address table that will serve as a look-up table during all further correction steps according to equations 1 and 2 . A distorted and a corrected DFTS-multiperspective image of a test grid are shown in Fig 7.

The real-time digital distortion correction unit consists of a 32-bit microprocessor system with three video-based random access memories (RAMs) as shown in the block diagram in Fig 8. One RAM is used as image memory for the digitized and distorted input image from the television chain. The second RAM provided the restortion corrected output image and serves as an additional image memory. The third RAM is used as an address memory storing the source addresses for each pixel of the output image. In addition, a program memory is available in the central processing unit to execute the geometric correction step and to determine the distortion parameters in the initial gauging phase as described previously.

The correction is performed while copying the input image onto the output image, by scanning the output image memory and the address memory simultaneously. Each pixel of the output image is taken from that location in the input image that has been looked up from the address memory. For this reason, no arith-
Fig 5. Pseudomask technique. Angiogram of a left coronary artery: native image (A), generated mask (B), and the resulting image after subtraction (C).

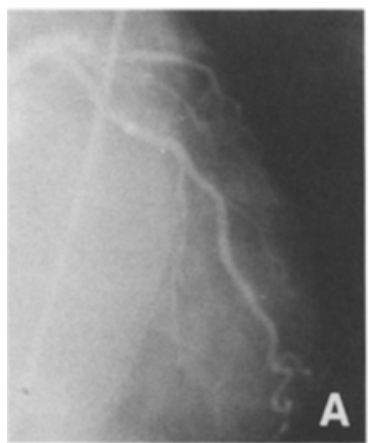

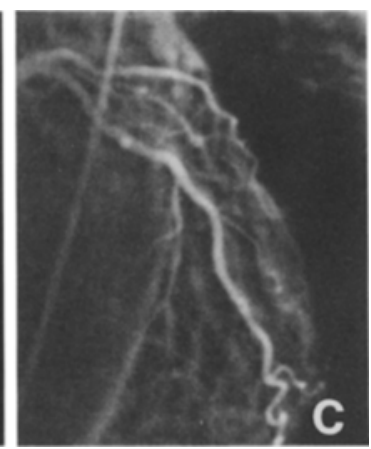




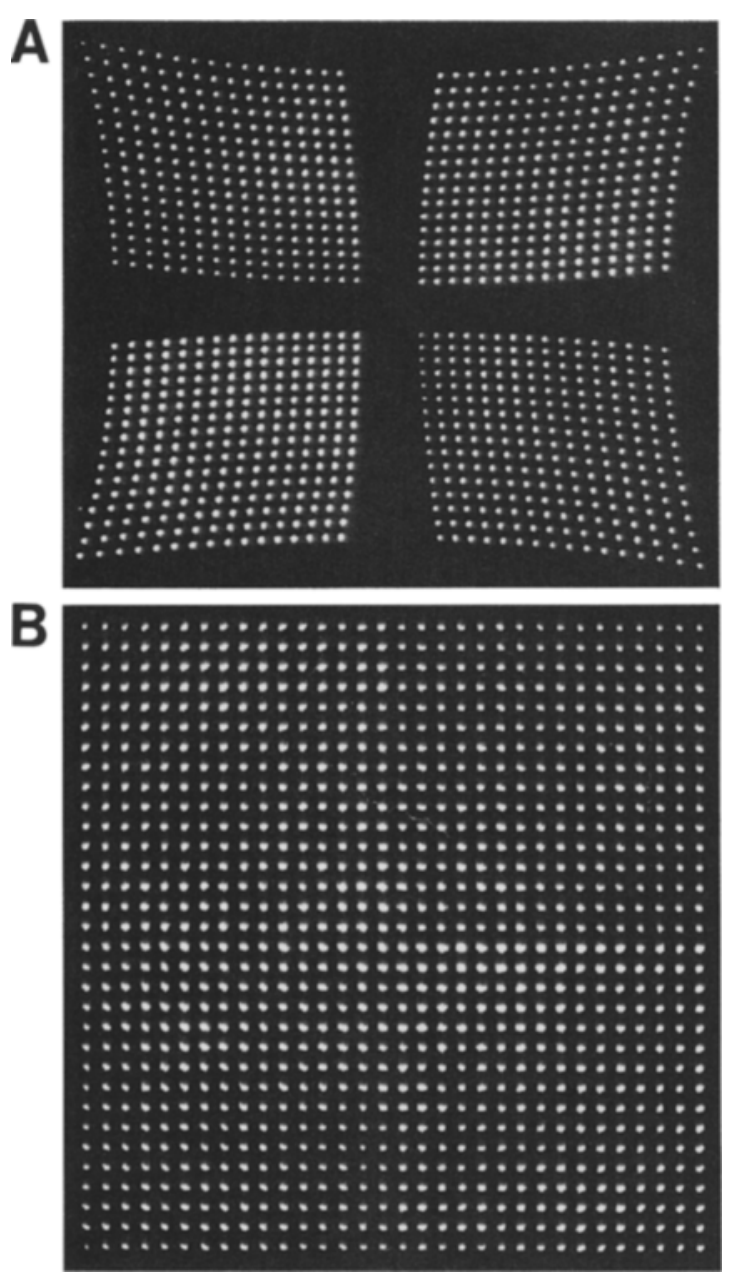

Fig 7. Distortion correction: distorted image (A) and corrected image (B) of the test grid.

metic operations are required during the correction step and, thus, even large image matrices can be corrected relatively fast using any commercial microprocessor system, eg, a 32-bit Motorola $680 \mathrm{X} 0$ microprocessor. ${ }^{14}$ The execution time is reduced to 100 to 300 milliseconds per $512 \times 512 \times 8$-bit image.

Two $512 \times 512 \times 8$-bit matrices for the input and output images require a storage capacity of 0.5 Mbyte. For a direct memory mapping, the addresses must be 20 bits wide and a storage capacity of 1 Mbyte is required..$^{14}$

\section{D RECONSTRUCTION METHOD}

DFTS is based on a nonlinear reconstruction algorithm, which produces significantly less artifacts for diluted objects than convential backprojection methods. ${ }^{15,16}$ Diluted objects are characterized by the fact that most of their volume elements (voxels) are empty. Coronary arteries filled with contrast medium approximate this condition if the background is subtracted, eg, by digital subtraction angiography. ${ }^{12}$ In this case, tomosynthesis can be performed with only few projections. The elimination of artifacts allows tomosynthesis to sum up slices to a DFTSmultiangiogram or to a new synthetic projection that can be combined with the original or another synthetic projection for a synthetic stereo view.

\section{Recording Geometry}

The imaged object is placed between two parallel planes, $R_{\mathrm{x}}$ and $R_{\mathrm{j}}$ (Fig 9). The x-ray tubes $X_{1}$, through $X_{\mathrm{k}}$ are positioned in the plane $R_{\mathrm{x}}$ and the recorded projections $B_{1}$ through $B_{\mathrm{k}}$ in the plane $R_{\mathrm{j}}$. A voxel $V_{\mathrm{m}}$ in layer $L_{\mathrm{m}}(1 \leq \mathrm{m} \leq$ $\mathrm{n}$ ) is projected into a pixel $P_{\mathrm{k}}$ of every projection $B_{\mathrm{k}}(1 \leq \mathrm{k} \leq \mathrm{k})$. Each pixel is a superposition of the projections of all other voxels on the corresponding ray:

$$
\mathrm{P}_{\mathrm{k}}=\sum_{\nu=1}^{\mathrm{n}} \mathrm{V}_{\mathrm{k} \nu} ; \quad \mathrm{V}_{\mathrm{km}}=\mathrm{V}_{\mathrm{m}} ; \quad 1 \leq \mathrm{m} \leq \mathrm{n} .
$$

The physical interpretation is given by the following equation:

$$
P_{k}=-\ln \left(J / J_{0}\right)=\lim _{n \rightarrow \infty} \sum_{\nu=1}^{n} V_{k \nu},
$$

where $J_{0}$ and $J$ describe the incident and the measured intensity in the pixel $P_{\mathrm{k}}$, respectively, and $V_{\kappa \nu}$ denotes the absorption in this voxel.

\section{Extreme Value Reconstruction Algorithm}

The backprojection simply fills the voxel $V_{m}$ with the average gray level:

$$
\overline{\mathrm{V}}_{\mathrm{m}}=\frac{1}{\mathrm{k}} \sum_{\mathrm{k}=1}^{\mathrm{k}} \mathrm{P}_{\mathrm{k}} \text {. }
$$

Backprojection produces good reconstructions if a large number of projections can be obtained and the object is homogeneous.

For the vessel structure of the coronary arteries an extreme value reconstruction algorithm for nonlinear decoding was developed. This method fills the voxel $\mathrm{V}_{\mathrm{m}}$ with the minimum gray-level:

$$
\mathrm{V}_{\mathrm{m}}^{*}=\min _{\mathrm{k}=1}^{\mathrm{k}} \mathrm{P}_{\mathrm{k}}
$$




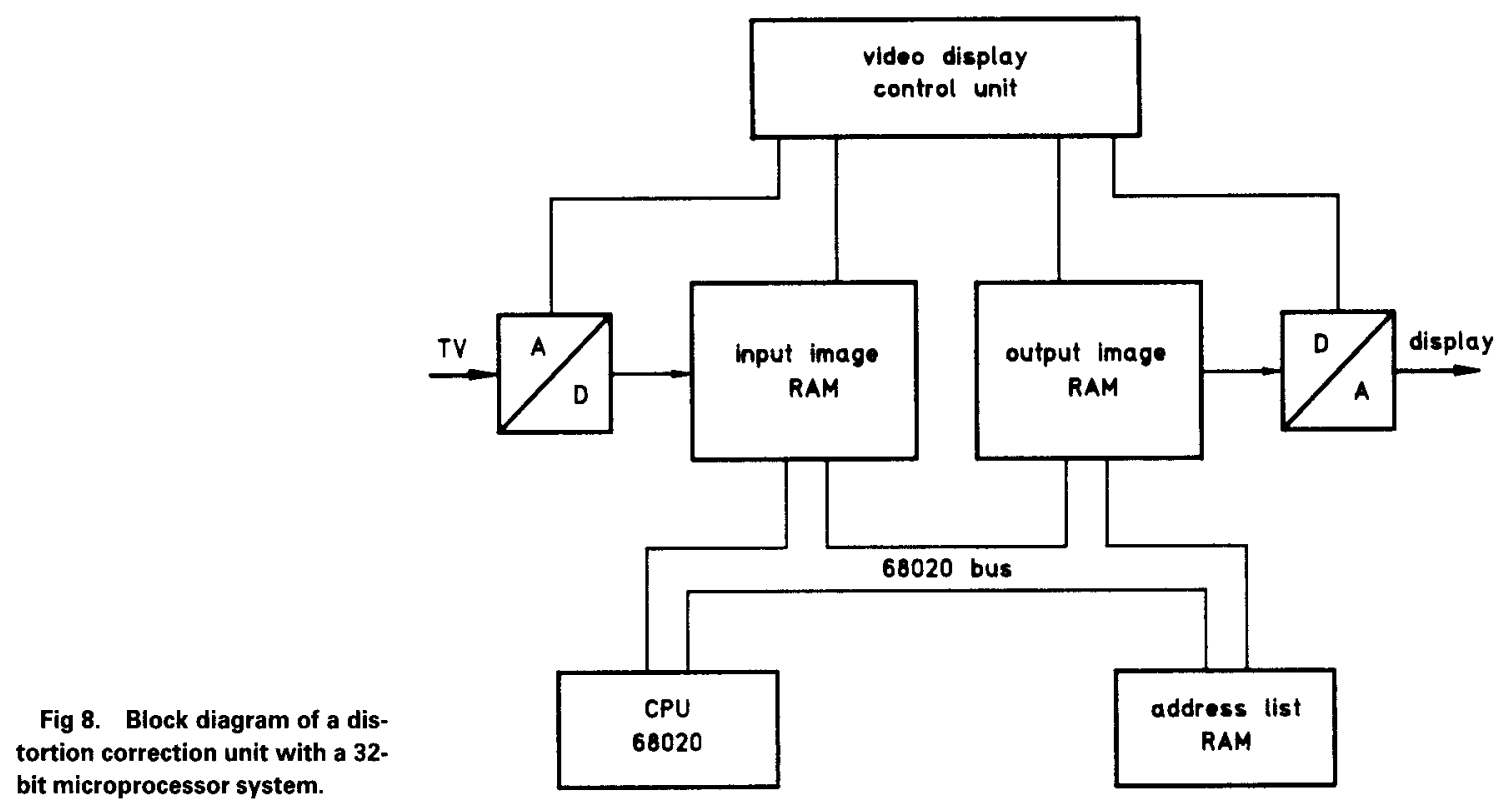

Good estimation of the gray level for a diluted object $O$ is given if:

$$
\mathrm{O} \leq \mathrm{V}_{\mathrm{m}} \leq \mathrm{V}_{\mathrm{m}}^{*} \leq \overline{\mathrm{V}}_{\mathrm{m}}
$$

The method is shown in a simple model in Fig 10 . The highly absorbing voxel S (streaky point) of the diluted object $O$ is projected from $X_{1}$ through $\mathrm{X}_{4}$-ray tubes in the source plane $\mathrm{R}_{\mathrm{x}}$ into the assigned pixels $\mathrm{P}_{1}$ through $\mathrm{P}_{4}$ in the image plane $R_{j}$. The projections $P_{1}, P_{2}$ and $P_{4}$ of voxel $S$ from the $\mathrm{X}$-ray tubes $\mathrm{X}_{1}, \mathrm{X}_{2}$ and $\mathrm{X}_{4}$ are overlaped by the projections of one or two voxels in other planes with relatively little absorption

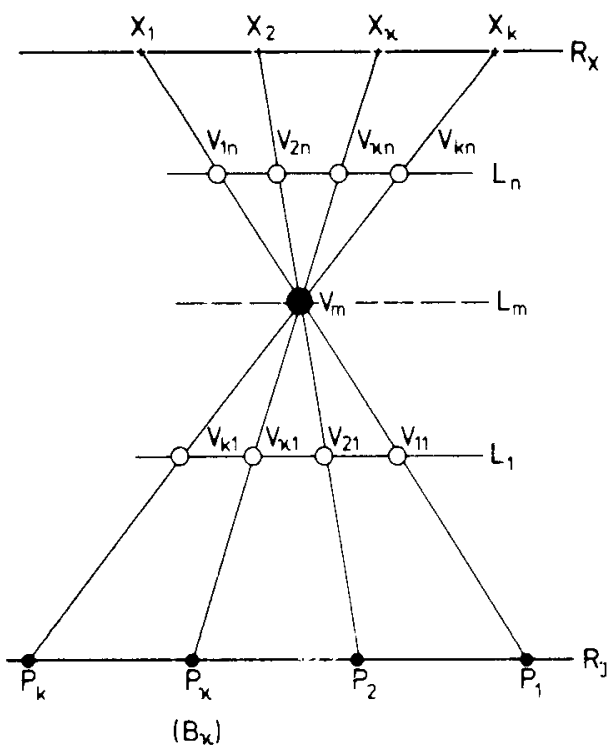

Fig 9. Projection of an arbitrary voxel $V_{m}$ from $k$ sources (streaky points). The projection $\mathrm{P}_{1}$ from $\mathrm{x}$-ray tube $X_{1}$ is covered by a voxel with high absorption (black point), whereas the projection $P_{3}$ from $x$-ray tube $X_{3}$ is devoid of any overlapping. The reconstruction of voxel $S$ by extreme-value decoding yields the pixel with the smallest gray level. In this example, pixel $\mathrm{P}_{3}$ represents the exact value of the voxel $S$.

With an earlier version of DFTS, using only four projections, it has been shown that this reconstruction method yields results of diagnostic image quality. ${ }^{10}$ However, artifacts are still

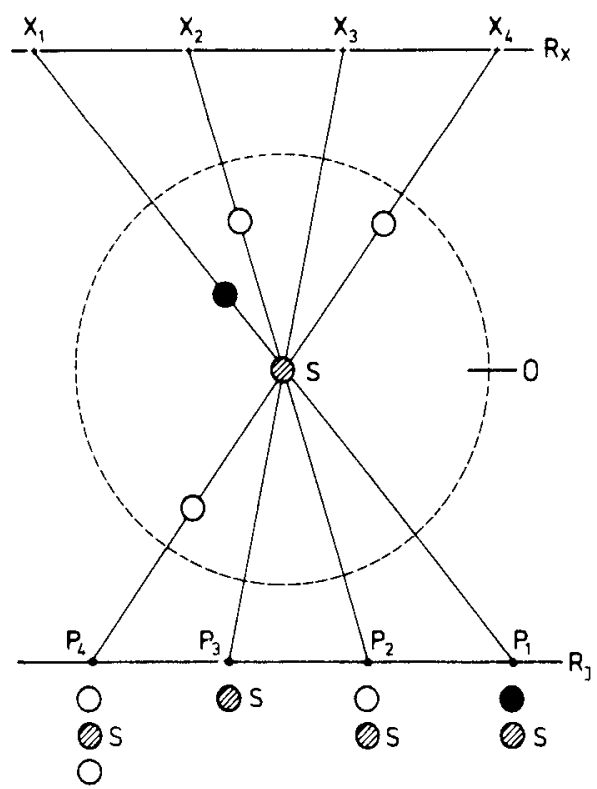

Fig 10. Principle of extreme value decoding by DFTS. 
present and they can be easily recognized using a rotating $3 \mathrm{D}$ image presentation. ${ }^{11}$

\section{Generation of Synthetic Projections}

For simplified explanation, only two $\mathrm{x}$-ray tubes, $X_{1}$ and $X_{2}$, are placed at a distance $D$ on plane $R_{x}$ as shown in Fig 11. We consider two bundles of rays coming from $X_{1}$ and $X_{2}$ which intersect in $R_{j}$ at a distance $H$ from $R_{x}$ in pixels of size $d$. The set of pixels defines the projection images $B_{1}$ and $B_{2}$. As a consequence of elementary geometric properties, all intersection points (voxels) of the two ray bundles are located on layers $L_{1}$, through $L_{n}$, called induced planes, parallel to $R_{x}$ and $R_{j}{ }^{17}$ The metric configuration of the induced planes is determined by the parameters $D, d$ and $H$. An arbitrary layer $L_{\mathrm{n}}$ has the distance:

$$
h_{n}(D)=\frac{H}{1+\frac{D}{n \cdot d}}
$$

from plane $R_{j}$. The distance between the voxels in layer $L_{n}$ is developed in the relation:

$$
d_{n}=\frac{d \cdot D}{D+n \cdot d} .
$$

The distance between the two pixels into which

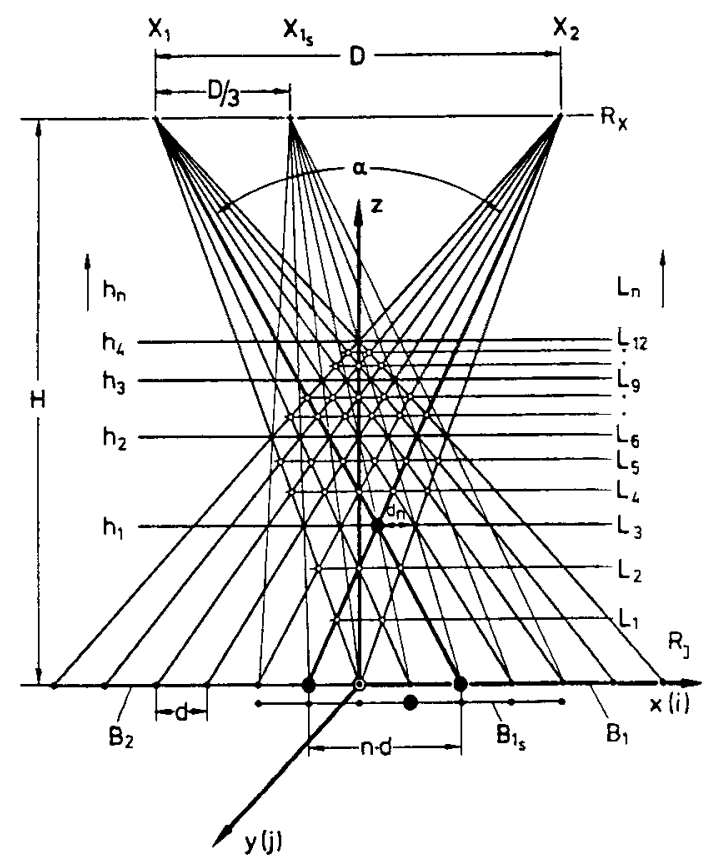

Fig 11. Principle of stereosynthesis by DFTS. an arbitrary voxel of $L_{n}(n=3$ in Fig 11) is projected will be $n \cdot d$. Therefore, the reconstruction of layer $L_{n}$ can be performed by shifting the projections $B_{1}$ and $B_{2}$ with respect to each other resulting in an image matrix and by applying equation 6 to each of the pixels of this image matrix. We assume that at the distance $D / m$ from the $\mathrm{x}$-ray tube $X_{1}$ an additional tube $X_{1_{s}}$ should be arranged (eg, $m=3$ ), that generates the projection image $B_{t_{s}} \cdot X_{l_{s}}$ induces with $X_{1}$ layers that matches only a subset of layers induced by $X_{1}$ and $X_{2}$. From equation 8 follows:

$$
\mathrm{h}_{\mathrm{n} \cdot \mathrm{m}}=\mathrm{h}_{\mathrm{n} \cdot \mathrm{m}}(\mathrm{D})=\mathrm{h}_{\mathrm{n}}(\mathrm{D} / \mathrm{m})=\mathrm{L}_{\mathrm{n}}
$$

This relationship means: the $n$th layer induced by $\mathrm{X}_{1}$ and $\mathrm{X}_{1 \mathrm{~s}}$ coincides with the $(n \cdot m)$ th layer induced by the original tubes $X_{1}$ and $X_{2}$ (eg, $\mathrm{h}_{1}=\mathrm{L}_{3}$ ).

Thus, for the calculation of the pseudoprojection $B_{1}$, the layers $L_{n}$ must be shifted by $n / m$ pixels. In the case of $\mathrm{m}=3$ the layers $\mathrm{L}_{3}, \mathrm{~L}_{6}, \mathrm{~L}_{9}$ and $L_{12}$ are used for synthesizing theprojection $B_{1_{s}}$. To suppress the background, each pixel $P_{1_{s}}$ of $B_{1_{s}}$ is the maximum of all voxels $V_{n}^{(1 s)}$ that are projected into this pixel (maximum-intensityprojection algorithm):

$$
\mathrm{P}_{1_{\mathrm{s}}}=\max _{v=1}^{\mathrm{n}}\left(\mathrm{V}_{v}^{\left(1_{\mathrm{s}}\right)}\right)
$$

\section{Measurement in Three Directions}

For a cartesian coordinating system (see Fig 11), equations 8 and 9 show the following for the metrical position:

$\mathrm{x}=\mathrm{i} \cdot \mathrm{d}_{\mathrm{n}} ; \quad \mathrm{y}=\mathrm{j} \cdot \mathrm{d}_{\mathrm{n}} ; \mathrm{z}=\mathrm{h}_{\mathrm{n}}$

where $i$ and $j$ denote the horizontal line and the vertical column indices in the image matrix, respectively. The accuracy of the depth measurement in the $z$ direction increases with the angle $\alpha$ between the projections $X_{1}$ and $X_{2}$.

\section{Personal Computer-Hosted Transputer Network for $3 D$ Reconstruction}

The pixel-oriented extreme-value algorithm is well suited for a parallel implementation on a personal computer-hosted transputer network (T-800, INMOS Ltd, Bristol, United Kingdom) as shown in Fig $12 .{ }^{18}$ First, for (parallel slice) reconstruction, the minimum value is chosen between projections $\mathrm{A}$ and $\mathrm{B}$ (node 1 and 2) and simultaneously between projection $\mathrm{C}$ and $\mathrm{D}$ (node 3 and 4). In a second step the 


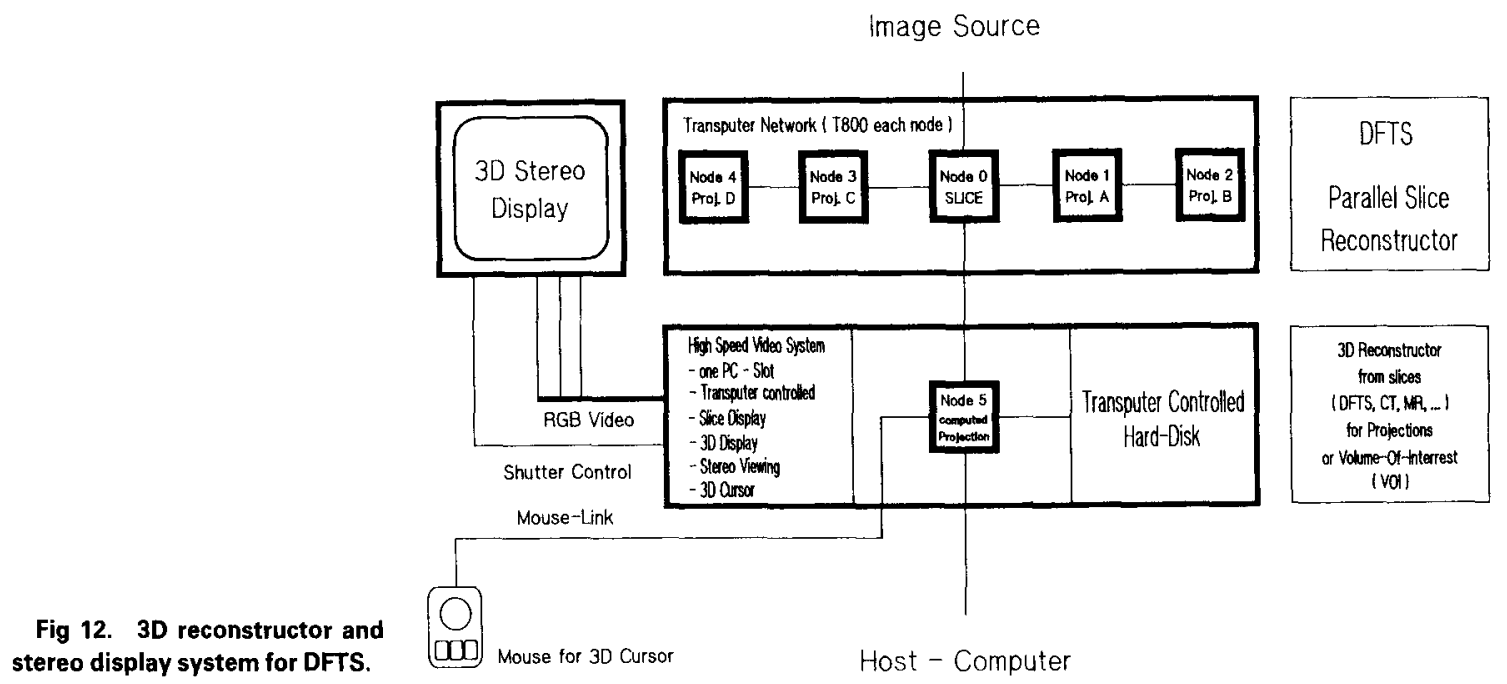

resulting signals are compared in node 0 for final determination of the minimum value. Nodes 0 and 5 are used to compute new projections using the maximum-intensity-projection algorithm. The computing time of a new projection ( $256 \times 256$ matrix) amounts to 15 seconds, which is tolerable for clinical use. The degree of parallelization can be further improved by partitioning the projections into subimages for even faster reconstruction.

\section{Rotating Presentation of 3D Data}

DFTS has the potential to obtain reconstructed 3D images of the coronary artery tree. Each coronary artery can be displayed in a rotating fashion about an arbitrary axis mimicking the third dimension by use of commercial software. A set of 20 reprojection images computed from a 3D data set spaced at intervals of $3^{\circ}$ is given in Fig 13 as an example of a clinical study. ${ }^{10}$ A continuous virtual rotation of the coronary artery tree is performed by displaying these images sequentially on a monitor screen. The 3D reconstruction of the ECG-synchronized dynamic tomoangiogram may also be presented and studied in similar fashion.

\section{Stereographic Presentation of $3 D$ Data}

The DFTS-multiangiogram can be combined with a complementary synthetic image to produce a stereoprojection. A Tektronix SGS 620 monitor (TEKTRONIX Inc, Beaverton, OR) combined with a liquid crystal shutter plate proved useful for the stereographic presenta- tion of that $3 \mathrm{D}$ information. It encodes the stereoprojections with right and left circular polarization. Special passive polarizing eyeglasses will provide a true 3D stereographic presentation. ${ }^{19}$ The effective vertical scan rate is $120 \mathrm{~Hz}$, enabling a flicker-free display. Two hardware cursors are combined as a stereo cursor controlled by a mouse for direct 3D interaction within the $3 \mathrm{D}$ data set to measure distances inside the arterial tree in the 3D space as shown in Fig 13.

\section{Quantitative Coronary Angiography}

An array of digital measuring functions is available for quantitative coronary angiography such as manually controlled stenosis measurement (caliper method) or automatic stenosis measurement with automated detection of both the vascular edges and the centerline of the vessel. In addition, an interactive contour correction, diameter statistics, as well as densitometric and hemodynamic analysis and the calculation of plaque mass are available. ${ }^{20}$

\section{Quantitative Ventriculography}

Manual and automatic determination of ejection fraction are available for quantitative analysis of left ventricular function. ${ }^{21,22}$ Analysis of wall motion is feasible by applying either the regional, centerline, or the Slager method. ${ }^{23-25}$

\section{Image Archiving}

A complete digital examination with DFTS including source and reconstructed images as well as processed images with results of a 


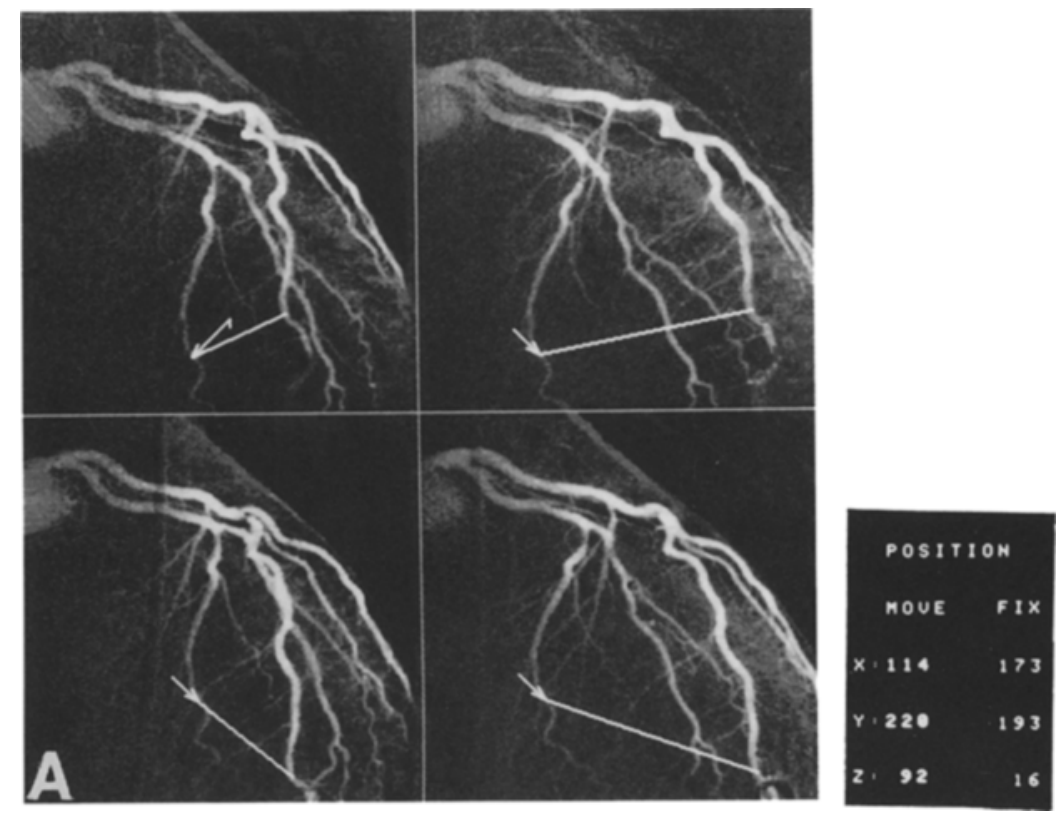

Fig 13. Stereographic presentation (original in left and right polarization) of a DFTS-multiangiogram of left coronary artery (A) and an example of 20 computed reprojection images spaced at intervals of $3^{\circ}$ used for continuous rotation by displaying these images sequentially on a monitor (B). Multiple-stereosynthesis: demonstration of a cursor-guided correct distance measurement in the 3D space (A).

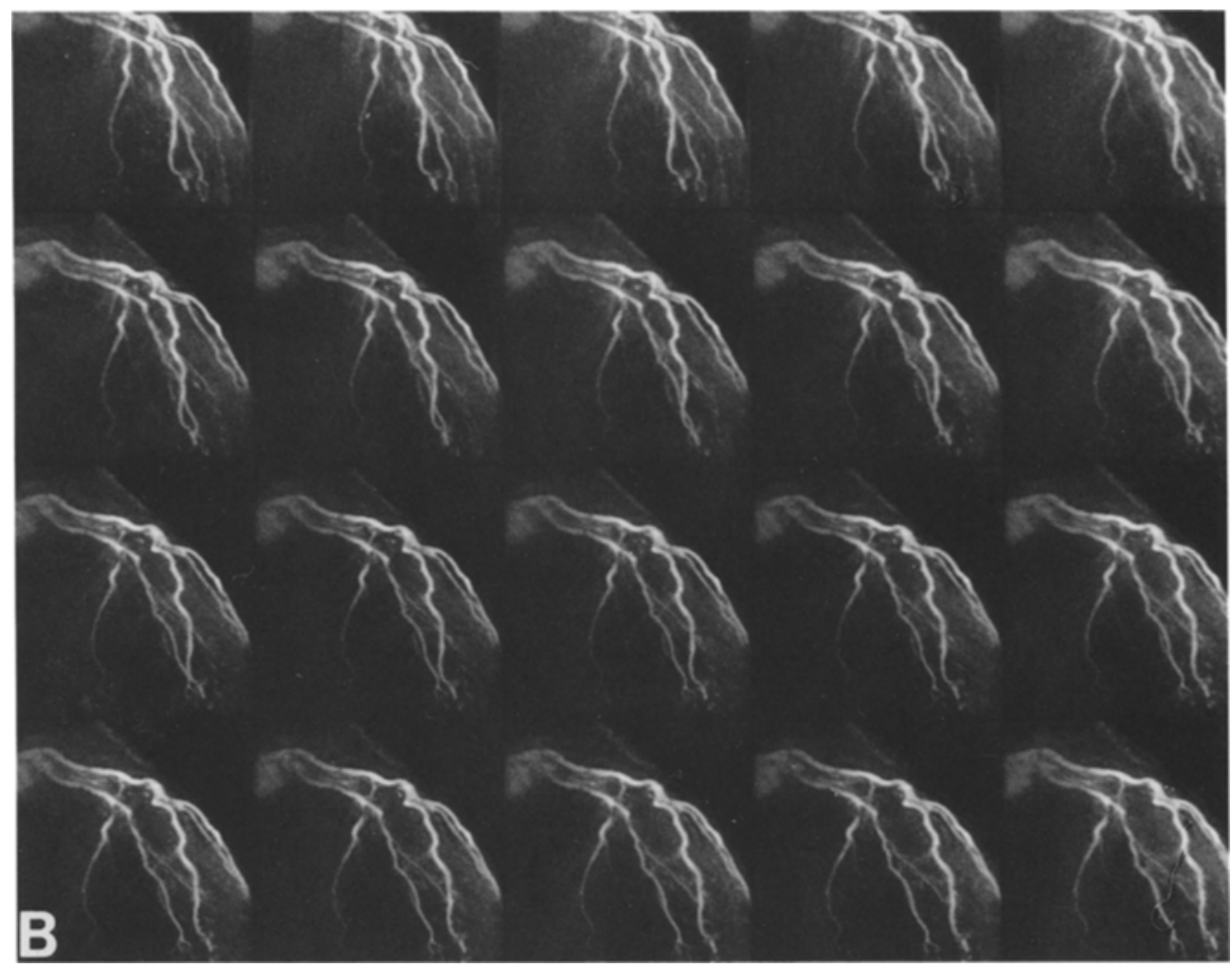

quantitative analysis and procedural data or, alternatively, the fully processed digital images can be archived on high-resolution S-VHS video tapes for reanalysis of data or for duplication. A separate archiving medium is installed for each patient. In addition, quantitative data on ventricular function and stenosis geometry may be recorded on a hard-copy imager such as a laser laser imager to fulfill legal requirements.

\section{EXPERIMENTAL STUDIES}

Postmortem coronary angiography of 30 human hearts with suspected CAD was performed with the $35-\mathrm{mm}$ cine technique and DFTS. ${ }^{11}$ 
The morphometric analysis was performed by the method of Brown et al. ${ }^{26}$ The angiographic percent diameter stenosis were calculated for 30 coronary lesions from the cineangiograms and the corresponding tomoangiograms as:

$\% \mathrm{DS}=\left(1-\frac{\text { stenotic diameter }}{\text { normal diameter }}\right) \times 100 \%$

The histologic measurement of the actual diameter stenosis was performed using identical formula. Regression analysis of angiographic measurements and histologic reference found similar results with coefficients of correlation in excess of $r=0.95(P<0.0001)$ as shown in Fig. 14. The standard error of estimation in measurements employing DFTS are slightly higher than by cine angiography; this could be a result of the lower resolution capacity in the current state of the system. The direct testing of the DFTS percent diameter stenosis with cine percent values do not differ significantly as shown in Fig 15.
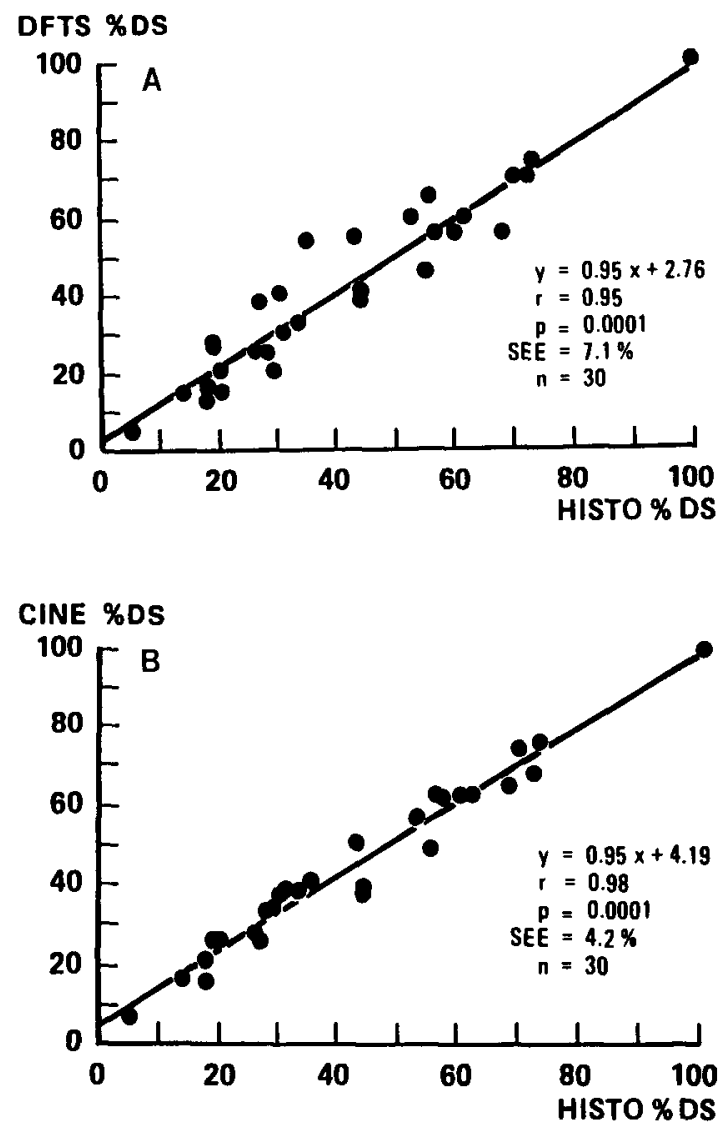

Fig 14. Comparison of the angiographic percent diameter stenosis by DFTS technique (DFTS \% DS) (A) and cine technique (CINE \% DS) (B) with the histologic reference (HISTO \% DS).

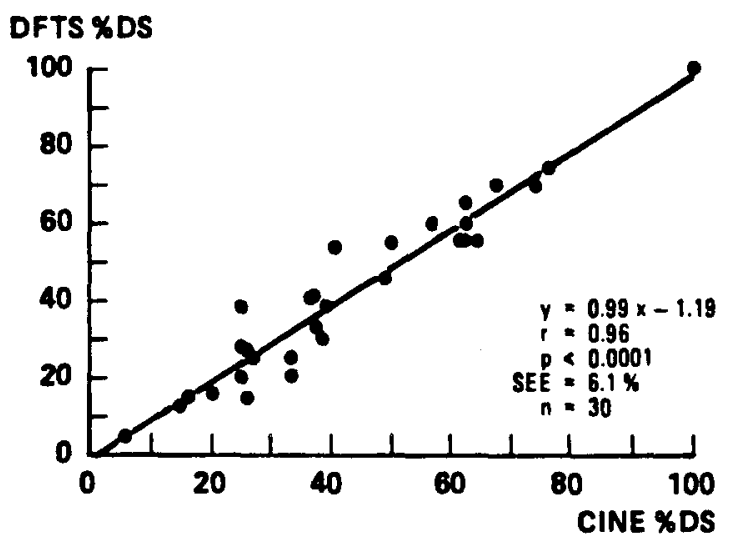

Fig 15. Comparison of the percent diameter stenosis by DFTS technique (DFTS \% DS) with cine technique [CINE \% DS).

\section{CONCLUSION}

The longitudinal assessment of the coronary pathology, ie, coronary atherosclerotic lesions is a prerequisite for the exact quantitative assessment of therapeutic interventions in modern cardiology. Not only interventions such as various forms of angioplasty, but also dietary and cholesterol-lowering drug-intervention trials have resulted in less progression or even regression in selected patients. ${ }^{27-29}$ However, to consolidate these encouraging results of new pharmacological intervention trials, a high volume of patients in large-scale trials must be screened and followed up regularly by angiography to show significant progression or regression of coronary lesions.

Thus, there is an urgent need for a reliable method: first, to assess the impact of the process of atherogenesis on the coronary artery lumen and on blood flow; second, to provide the image resolution and reproducibility required for quantitative coronary angiography; and third, to ensure safety, cost-effectiveness, and feasibility on an ambulatory basis for angiocardiographic screening. DFTS may provide these features in the near future, merging a rapid and highly reproducible outpatient coronary angiography setting with all prerequisites of quantitative angiography.

The potential sources of error related to quantitative angiography can also be avoided with DFTS. Once the magnification, tubevoltage, and frame exposure are optimized and set for best contrast, minimal quantum noise, and motion blur, the individual, patient-related, and procedure-related sources of error (or vari- 
ability) are recorded and controlled for follow-up studies. Methodological characteristics such as the motionless, fixed angiography table imply further conceptional advantages of DFTS ensuring optimal reproducibility for repeat angiographic studies. More importantly, the tomographic acquisition of the coronary tree in multiple layers of voxels allows DFTS to select the optimal angle of view for any given lesion of an individual patient. Once recorded, this setting can be easily reproduced in any future DFTS study. The routines for quantitative evaluation of stenoses (geometric and densitometric) can be easily applied to DFTS-tomoangiograms and executed with optimal reliability because even the procedural data are recorded.

Because DFTS can be performed safely in an ambulatory setting by the use of $5 \mathrm{~F}$ catheters and relatively small amounts of contrast medium, the prospect of screening patients at moderate or intermediate risk for coronary heart disease exists. Moreover, nonselective angiography using a specially designed catheter placed in ascending aorta will probably allow diagnostic imaging with further reduction of contrast medium, radiation dose, and time.

Therefore, as an ambulatory method with digital data storage and excellent reproducibility, DFTS may be helpful to address more demanding and currently unsolved problems. Regular DFTS studies of the coronary anatomy may elucidate whether progression of atherosclerosis is linear or episodic, whether accelerated progression can be tampered by interventions, and whether there are different patterns of progression or regression in CAD. In addition, the effects of aging may be separated from atherogenesis or from compensatory mechanisms. ${ }^{30}$ Thus, DFTS, in combination with quantitative analysis, will be a useful diagnostic tool to elucidate both the pathologic mechanism of coronary atherosclerotic progression or regression and the efficacy of various therapeutic interventions such as angioplastic procedures or lipid-lowering dietary interventions.

\section{REFERENCES}

1. Detre KM, Kelsey SF, Passamani ER, et al: Reliability of assessing change with sequential coronary angiography. Am Heart J 104:816-823, 1982

2. Reiber LHC, Serruys PW, Kooijman CJ, et al: Assessment of short-, medium,- and long-term variations in arterial dimensions from computer-assisted quantitation of coronary cineangiograms. Circulation 71:280-288, 1985

3. Klotz E, Weiss H: Three dimensional coded aperture imaging using nonredundant point distributions. Optics Comm 11:368-372, 1974

4. Weiss $\mathrm{H}$, Klotz E, Linde L: Deconvolution systems for coded aperture images of three-dimensional X-ray objects. Optics Laser Technol 6:117-120, 1975

5. Weiss H, Klotz E, Linde R, et al: Coded aperture imaging with $\mathrm{X}$-rays (flashing tomosynthesis). Opt Acta 24:305-325, 1977

6. Nadjmi M, Weiss H, Klotz E, et al: Flashing tomosyntheis-a new tomographic method. Neuroradiology 19:113117,1980

7. Nadjmi M, Weiss H, Klotz E, et al: Flashing tomosynthesis: First clinical results. Medicamundi 25:9-17, 1980

8. Woelke $\mathrm{H}$, Hanrath $\mathrm{P}$, Schlüter $\mathrm{M}$, et al: Work in progress. Flashing tomosynthesis: A tomograhpic technique for quantitative coronary angiography. Radiology 145:357360, 1982

9. Becher H, Schlüter M, Mathey DG, et al: Coronary angiography with flashing tomosynthesis. Eur Heart J 6:399408,1985

10. Haaker P, Klotz E, Koppe R, et al: First clinical results with digital flashing tomosynthesis in coronary angiography. Eur Heart J 6:913-920, 1985

11. Stiel GM, Stiel LSG, Donath K, et al: Digital flashing tomosynthesis (DFTS)-a technique for three-dimensional coronary angiography. Int J Card Imag 5:53-61, 1989

12. Mistretta CA: Digital subtraction angiography. The shape of things to come. Diagn Imag 130:36-40, 1982

13. Devijver PA, Ronse C, Haaker P, et al: Pseudomask technique for digital subtraction angiography. InformatikFachberichte, in Proceedings of DAGM/ÖAGM Symposium Musterekennung 1984. Berlin, Germany, SpringerVerlag, 1984, pp 230-236

14. Haaker P, Klotz E, Koppe R, et al: Real-time distortion correction of digital X-ray II/TV-systems: An application example for digital flashing tomosynthesis (DFTS). Int J Card Imag 6:39-45, 1990/91

15. Haaker P, Klotz E, Koppe R, et al: A new digital tomosynthesis method with less artifacts for angiography. Med Phys 12:431-436, 1985

16. Klotz E, Haaker P, Koppe R, et al: Three-dimensional angiography with digital flashing tomosynthesis. SPIE 804:306-313, 1987

17. Hamasaki J, Yokota K: Direct recording and reconstruction of 3-D X-ray images. Appl. Optics 17:3125-3132, 1978

18. Klotz E, Haaker P, Koppe R, et al: 3D reconstruction of vascular structures from few X-ray projections, in Lemke HU, Rhodes ML, Jaffe CC, et al (eds): Proceedings of the International Symposium CAR'91. Berlin, Germany, Springer-Verlag, 1991, pp 55-61

19. Koppe R, Haaker P, Klotz E, et al: Interactive stereotactic procedures with digital flashing tomosynthesis, in Lemke HU, Rhodes ML, Jaffe CC, et al (eds): Proceedings of the International Symposium CAR' 87 . Berlin, Germany, Springer-Verlag, 1987, pp 359-368 
20. Reiber JHC, Kooijman CJ, Slager CJ: Improved densitometric assessment of percent area-stenosis from coronary cineangiograms. Advances in image processing. Proc SPIE 804:152-158, 1987

21. Sandler $H$, Dodge HT: The use of single plane angiocardiograms for the calculation of left ventricular volume in man. Am Heart J 75:325-334, 1968

22. van Leeuwen PJ, Reiber JHC: Left ventricular contour detection with a dynamic search algorithm. Advances in image processing. Proc SPIE 804:131-135, 1987

23. Wong WH, Kirkeeide RL, Gould KL: Computer applications in angiography, in Collins SM, Skorton DJ (eds): Cardiac Imaging and Image Processing. New York, NY, McGraw-Hill, 1986, pp 232-233

24. Sheehan FH, Bolson EL, Dodge HT, et al: Advantages and applications of the centerline method for characterizing regional ventricular function. Circulation 74:293305,1986

25. Slager CJ, Hooghoudt TEH, Serruys PW, et al; Quantitative assessment of regional left ventricular motion using endocardial landmarks. J Am Coll Cardiol 7:317-326, 1986

26. Brown BG, Bolson E, Frimer M, et al: Quantitative coronary arteriography. Estimation of dimensions, hemodynamic resistance, and atheroma mass of coronary artery lesions using arteriogram and digital computation. Circulation 55:329-337, 1977

27. The Lipid Clinics Coronary Primary Prevention Trial Results: 1. Reduction in incidence of coronary heart disease. JAMA 251:351-374, 1984

28. Brown G, Albers J, Fisher LD, et al: Regression of coronary artery disease as a result of intensive lipid lowering therapy in men with high levels of apolipoprotein $\mathrm{B}$. N Engl J Med 323:1289-1298, 1990

29. Ornisch O, Brown SE, Scherwitz LW, et al: Can lifestyle changes reverse coronary heart disease? The Lifestyle Heart Trial. Lancet 336:129-133, 1990

30. Stiel GM, Stiel LSG, Schofer J, et al: Impact of compensatory enlargement of atherosclerotic coronary arteries on angiographic assessment of coronary heart disease. Circulation 80:1603-1609, 1989 